\title{
SOLVING LINEAR FREDHOLM INTEGRO-DIFFERENTIAL EQUATION BY NYSTRÖM METHOD
}

\author{
Boutheina Tair, Hamza Guebbai, Sami Segni, Mourad Ghiat \\ Laboratoire des Mathématiques Appliquées et Modélisation,Université 8 Mai 1945 \\ Guelma 24000, Algeria \\ tair.boutheina@univ-guelma.dz,tairboutheina2@gmail.com \\ guebaihamza@yahoo.fr,hamza.guebbai@univ-guelma.dz \\ segnianis@gmail.com \\ mourad.ghi24@gmail.com
}

Received: 29 March 2021; Accepted: 3 August 2021

\begin{abstract}
The study of the solution's existence and uniqueness for the linear integrodifferential Fredholm equation and the application of the Nyström method to approximate the solution is what we will present in this paper. We use the Neumann theorem to construct a sufficient condition that ensures the solution's existence and uniqueness of our problem in the Banach space $C^{1}[a, b]$. We have applied the Nyström method based on the trapezoidal rule to avoid adding other conditions in order to the approximation method's convergence. The Nyström method discretizes the integro-differential equation into solving a linear system. Only with the existence and uniqueness condition, we show the solution's existence and uniqueness of the linear system and the convergence of the numerical solution to the exact solution in infinite norm sense. We present two theorems to give a good estimate of the error. Also, to show the efficiency and accuracy of the Nyström method, some numerical examples will be provided at the end of this work.
\end{abstract}

MSC 2010: 45B05, 45J05, 47G20, 34K28, 45L05, 65R20

Keywords: Fredholm integral equation, system of integral equations, integro-differential equations, Nyström method

\section{Introduction}

The importance of the integro-differential equations is clearly demonstrated in physics, mathematical modelling, chemistry, biology, engineering, etc. [1-4]. This has led to the presence of different types and forms of equations. Therefore, an approximation and numerical processing is necessary. Most of the equations found, used and studied are the equations where the derivative of the unknown function is outside the integral sign, and scientists have built several methods to estimate the solution like the Homotopy perturbation method [5], Fractional order operational matrix methods the Adomain decomposition method [6] and another method using Legendre scaling functions [7,8], Embedded pseudo-Runge-Kutta methods [9], 
Volterra-Runge-Kutta method [10], the iterative variational method [11] and the projection methods [12].

Contrary to existing reasearch, the numerical method that we will present in this article is the Nyström method. However, this method was applied in the first state to approximate the solution of an integral equation and its theoretical framework is well given by Atkinson and Han in [13]. Recently, many others have relied on this method such as H. Guebbai et al. in [14, 15], H. Zhou and Q. Wang [16], S. Segni et al. [17], M. Ghiat et al. [18], S. Salah et al. [19], Lemita and Guebbai [20]: to give an estimation solution of the non-linear integro-differential Volterra equation with a continuous kernel, or a weakly singular kernel [21,22] or to approximate a solution of a Fredholm linear system.

Our objective is to treat a new type of Fredholm integro-differential equation where the derivative is inside the integral sign. We construct an approximate solution based on the Nyström method, especially since this method in general is used for solving integral equations numerically. We focused on the study of this equation on two sides; the analytical and numerical side.

Let $u \in C^{1}[a, b]$ be a solution of the following linear Fredholm integro-differential equation:

$$
\forall x \in[a, b], \lambda u(x)=\int_{a}^{b} K_{1}(x, t) u(t) d t+\int_{a}^{b} K_{2}(x, t) u^{\prime}(t) d t+f(x),
$$

where $\lambda \neq 0$ is a complex parameter, $f \in C^{1}[a, b]$ and $K_{i}$, for $i=1,2$ are given functions. We construct some conditions that ensures solution's existence and uniqueness of equation (1). We prove that those conditions are compatible with the Nystöme approximation method.

\section{Analytical study}

In the starting point of our work, we construct a condition that ensures the existence and uniqueness of the solution. Let $X=C^{1}[a, b]$ be a Banach space with the norm:

$$
\forall v \in X, \quad\|v\|_{X}=\max _{a \leq x \leq b}|v(x)|+\max _{a \leq x \leq b}\left|v^{\prime}(x)\right| .
$$

We suppose that $K_{i}$, for $i=1,2$ satisfied the next assumption:

$$
\left(H_{1}\right) \| \begin{gathered}
\frac{\partial K_{i}}{\partial x}(x, t) \in C^{0}\left([a, b]^{2}, \mathbb{R}\right), \\
\max _{a \leq x, t \leq b}\left(\left|K_{i}(x, t)\right|,\left|\frac{\partial K}{\partial x_{i}}(x, t)\right|\right) \leq M_{i} .
\end{gathered}
$$


Using this assumption to define the linear operator $T$ by:

$$
\begin{aligned}
T: X & \longrightarrow X \\
v & \longmapsto T v(x)=\int_{a}^{b} K_{1}(x, t) v(t) d t+\int_{a}^{b} K_{2}(x, t) v^{\prime}(t) d t, \quad \forall x \in[a, b] .
\end{aligned}
$$

It is clear that,

$$
\forall x \in[a, b],(T v)^{\prime}(x)=\int_{a}^{b} \frac{\partial K_{1}}{\partial x}(x, t) v(t) d t+\int_{a}^{b} \frac{\partial K_{2}}{\partial x}(x, t) v^{\prime}(t) d t
$$

Then, the derivative $u^{\prime}$ is given implicitly by

$$
\forall x \in[a, b], \lambda u^{\prime}(x)=\int_{a}^{b} \frac{\partial K_{1}}{\partial x}(x, t) u(t) d t+\int_{a}^{b} \frac{\partial K_{2}}{\partial x}(x, t) u^{\prime}(t) d t+f^{\prime}(x) .
$$

Theorem 1 Under the assumption $\left(H_{1}\right)$ and if $|\lambda|>2(b-a)\left(M_{1}+M_{2}\right)$, then the equation (1) has a unique solution in $X$.

PROOF To prove the solution's existence and uniqueness for (1), we write it under the form

$$
(\lambda I-T) u=f .
$$

We will demonstrate that $(\lambda I-T)^{-1}$ exists and is bounded. First of all, we define $\|T\|$ by

$$
\|T\|=\sup _{\|u\|_{X} \leq 1}\|T u\|_{X}
$$

Such that,

$$
\|T u\|_{X}=\max _{a \leq x \leq b}|T u(x)|+\max _{a \leq x \leq b}\left|(T u)^{\prime}(x)\right| .
$$

However, we have

$$
\begin{aligned}
|T u(x)| & \leq \int_{a}^{b}\left|K_{1}(x, t)\right||u(t)| d t+\int_{a}^{b}\left|K_{2}(x, t)\right|\left|u^{\prime}(t)\right| d t, \\
& \leq(b-a) M_{1} \max _{a \leq t \leq b}|u(t)|+(b-a) M_{2} \max _{a \leq t \leq b}\left|u^{\prime}(t)\right|, \\
& \leq(b-a)\left(M_{1}+M_{2}\right)\|u\|_{X},
\end{aligned}
$$

and

$$
\begin{aligned}
\left|(T u)^{\prime}(x)\right| & \leq \int_{a}^{b}\left|\frac{\partial K_{1}}{\partial x}(x, t)\right||u(t)| d t+\int_{a}^{b}\left|\frac{\partial K_{2}}{\partial x}(x, t)\right|\left|u^{\prime}(t)\right| d t \\
& \leq(b-a) M_{1} \max _{a \leq t \leq b}|u(t)|+(b-a) M_{2} \max _{a \leq t \leq b}\left|u^{\prime}(t)\right|, \\
& \leq(b-a)\left(M_{1}+M_{2}\right)\|u\|_{X} .
\end{aligned}
$$


Substituting (8) and (9) in (7), we obtain

$$
\|T u\|_{X} \leq 2(b-a)\left(M_{1}+M_{2}\right)\|u\|_{X},
$$

which implies that,

$$
\|T\| \leq 2(b-a)\left(M_{1}+M_{2}\right) .
$$

Using the fact that $|\lambda|>2(b-a)\left(M_{1}+M_{2}\right)$, we get $\| T||<|\lambda|$. Therefore, according to theorem 1.2.11 in [23] $s p(T) \subseteq \overline{B(0,\|T\|)}$, where $s p(T)$ is the spectrum set of $T$. Then

$$
\left\{\lambda \in \mathbb{C}^{*},|\lambda|>2(b-a)\left(M_{1}+M_{2}\right)\right\} \subseteq r e(T),
$$

where, $r e(T)$ is the resolvant set of $T$. We select the Nuemmann's theorem [24] to confirm that $(\lambda I-t)^{-1}$ exists and

\section{Numerical study}

$$
\left\|(\lambda I-T)^{-1}\right\| \leq \frac{1}{|\lambda|-|| T||} \leq \frac{1}{|\lambda|-2(b-a)\left(M_{1}+M_{2}\right)} .
$$

To search for an approximation solution of the system (1) and (4), we use the Nyström method which is based on the following numerical integration scheme:

$$
\forall n \geq 1, \forall \phi \in C^{0}[a, b], \quad \int_{a}^{b} \phi(x) d x \approx \sum_{i=0}^{n} \omega_{i} \phi\left(x_{i}\right),
$$

where, $\left\{\omega_{i}\right\}_{0 \leq i \leq n}$ called quadrature weights such that $W=\sup _{n \geq 1} \sum_{i=0}^{n}\left|\omega_{i}\right|<\infty$, $h=\frac{(b-a)}{n}$ and $x_{i}=a+i h$, for $i=0,1,2, \ldots, n$, are points discretization of the interval $[a, b]$.

Applying (13) to the integral term of the system (1)-(4), we obtain

$$
\left\{\begin{array}{l}
\lambda u(x)=\sum_{j=0}^{n} \omega_{j} K_{1}\left(x, x_{j}\right) u\left(x_{j}\right)+\sum_{j=0}^{n} \omega_{j} K_{2}\left(x, x_{j}\right) u^{\prime}\left(x_{j}\right)+f(x)+e_{1, n}(x), \\
\lambda u^{\prime}(x)=\sum_{j=0}^{n} \omega_{j} \frac{\partial K_{1}}{\partial x}\left(x, x_{j}\right) u\left(x_{j}\right)+\sum_{j=0}^{n} \omega_{j} \frac{\partial K_{2}}{\partial x}\left(x, x_{j}\right) u^{\prime}\left(x_{j}\right)+f^{\prime}(x)+e_{2, n}(x),
\end{array}\right.
$$

where, local errors are given by:

$$
\begin{aligned}
e_{1, n}(x) & =\int_{a}^{b}\left[K_{1}(x, t) u(t)+K_{2}(x, t) u^{\prime}(t)\right] d t \\
& -\sum_{j=0}^{n} \omega_{j}\left[K_{1}\left(x, x_{j}\right) u\left(x_{j}\right)-K_{2}\left(x, x_{j}\right) u^{\prime}\left(x_{j}\right)\right],
\end{aligned}
$$




$$
\begin{aligned}
e_{2, n}(x) & =\int_{a}^{b}\left[\frac{\partial K_{1}}{\partial x}(x, t) u(t)+\frac{\partial K_{2}}{\partial x}(x, t) u^{\prime}(t)\right] d t \\
& -\sum_{j=0}^{n} \omega_{j}\left[\frac{\partial K_{1}}{\partial x}\left(x, x_{j}\right) u\left(x_{j}\right)-\frac{\partial K_{2}}{\partial x}\left(x, x_{j}\right) u^{\prime}\left(x_{j}\right)\right] .
\end{aligned}
$$

We set $x=x_{i}$ and we suppose that $\forall x \in[a, b],\left\{e_{p, n}(x)\right\}_{1 \leq p \leq 2}$ are negligible to get the following linear approximation system with $2 n+2$ equations:

$$
\left\{\begin{array}{l}
\lambda u_{i}=\sum_{j=0}^{n} \omega_{j} K_{1}\left(x_{i}, x_{j}\right) u_{j}+\sum_{j=0}^{n} \omega_{j} K_{2}\left(x_{i}, x_{j}\right) u_{j}^{\prime}+f_{i}, \\
\lambda u_{i}^{\prime}=\sum_{j=0}^{n} \omega_{j} \frac{\partial K_{1}}{\partial x}\left(x_{i}, x_{j}\right) u_{j}+\sum_{j=0}^{n} \omega_{j} \frac{\partial K_{2}}{\partial x}\left(x_{i}, x_{j}\right) u_{j}^{\prime}+f_{j}^{\prime},
\end{array}\right.
$$

where $f_{i}=f\left(x_{i}\right), f_{i}^{\prime}=f^{\prime}\left(x_{i}\right)$ and $u_{i}, u_{i}^{\prime}$ are the approximations of $u\left(x_{i}\right)$ and $u^{\prime}\left(x_{i}\right)$ respectively.

\subsection{System analysis}

Theorem 2 If $|\lambda|>2 W\left(M_{1}+M_{2}\right)$, then the system (16) has a unique solution.

PROOF To demonstrate the existence of the system solution (16), we apply the Banach fixed point theorem. For this reason, we write the system (16) in the following form:

$$
U=A[U]
$$

where, $U=\left(u_{0}, u_{1}, \ldots, u_{n}, u_{0}^{\prime}, u_{1}^{\prime}, \ldots, u_{n}^{\prime}\right)^{t}$ is a vector of $\mathbb{R}^{2 n+2}$ and

$$
A[U]=\left\{\begin{array}{l}
\frac{1}{\lambda}\left[\sum_{j=0}^{n} \omega_{j} K_{1}\left(x_{i}, x_{j}\right) u_{j}+\sum_{j=0}^{n} \omega_{j} K_{2}\left(x_{i}, x_{j}\right) u_{j}^{\prime}+f_{i}\right], \quad 0 \leq i \leq n, \\
\frac{1}{\lambda}\left[\sum_{j=0}^{n} \omega_{j} \frac{\partial K_{1}}{\partial x}\left(x_{i-n-1}, x_{j}\right) u_{j}+\sum_{j=0}^{n} \omega_{j} \frac{\partial K_{2}}{\partial x}\left(x_{i-n-1}, x_{j}\right) u_{j}^{\prime}+f_{i}^{\prime}\right], n+1 \leq i \leq 2 n+2 .
\end{array}\right.
$$

For $\mathbb{R}^{2 n+2}$, we use the following norm:

$$
\|U\|_{\mathbb{R}^{2 n+2}}=\max _{0 \leq i \leq n}\left|u_{i}\right|+\max _{0 \leq i \leq n}\left|u_{i}^{\prime}\right| .
$$

Let $U$ and $V \in \mathbb{R}^{2 n+2}$, then

$$
\|A[U]-A[V]\|_{\mathbb{R}^{2 n+2}} \leq \frac{2 W\left(M_{1}+M_{2}\right)}{|\lambda|}\|U-V\|_{\mathbb{R}^{2 n+2}}
$$


Using the fact that $|\lambda|>2 W\left(M_{1}+M_{2}\right)$ and the Banach fixed point theorem, we conclude that the system (16) has a unique solution.

\subsection{Error analysis}

Our goal is to choose a numerical quadrature that provides that the error estimation converges to 0 . We select the formula of trapezoidal rules to ensure this convergence, see [13] and [25]. It also provides $\sum_{j=0}^{n}\left|\omega_{j}\right|=(b-a)$, which makes the analytical and numerical study compatible. In this rule, we have $\forall n \geq 1$

$$
\| \begin{aligned}
& h=\frac{(b-a)}{n}, \\
& \omega_{0}=\omega_{n}=\frac{h}{2}, \\
& \omega_{1}=\omega_{2}=\cdots=\omega_{n-1}=h, \\
& x_{i}=a+(i-1) h, i=0,1, \ldots, n .
\end{aligned}
$$

First, we define the continuity module $\kappa_{0}$ by

$$
\forall h>0, \forall v \in C^{0}[a, b], \kappa_{0}(v, h)=\sup _{|x-y| \leq h}|v(x)-v(y)|,
$$

and the continuity module $\kappa_{1}$ as follows

$$
\forall h>0, \forall v \in C^{1}[a, b], \kappa_{1}(v, h)=\kappa_{0}(v, h)+\kappa_{0}\left(v^{\prime}, h\right),
$$

Theorem 3 Let $e_{n}=\left(e_{1, n}\left(x_{0}\right), e_{1, n}\left(x_{1}\right), \ldots, e_{1, n}\left(x_{n}\right), e_{2, n}\left(x_{0}\right), e_{2, n}\left(x_{1}\right), \ldots, e_{2, n}\left(x_{n}\right)\right)^{t}$ be a vector in $\mathbb{R}^{2 n+2}$, then:

$$
\left\|e_{n}\right\|_{\mathbb{R}^{2 n+2}} \leq(b-a) \sum_{p=1}^{2} M_{p}\left[\max _{0 \leq i \leq n} \kappa_{1}\left(K_{p_{x_{i}}}, h\right)\|u\|_{X}+2 \kappa_{1}(u, h)\right],
$$

where, $K_{p_{x_{i}}}=K_{p}\left(x_{i},.\right)$, for $p=1,2$ and $i=0,1, \ldots, n$.

PROOF For $i=0,1, \ldots, n$, we have

$$
\begin{aligned}
\left|e_{1, n}\left(x_{i}\right)\right| & =\mid \sum_{j=0}^{n-1} \int_{x_{j}}^{x_{j+1}} K_{1}\left(x_{i}, t\right) u(t) d t-\frac{h}{2}\left[K_{1}\left(x_{i}, x_{j+1}\right) u\left(x_{j+1}\right)+K_{1}\left(x_{i}, x_{j}\right) u\left(x_{j}\right)\right] \\
& +\int_{x_{j}}^{x_{j+1}} K_{2}\left(x_{i}, t\right) u^{\prime}(t) d t-\frac{h}{2}\left[K_{2}\left(x_{i}, x_{j+1}\right) u^{\prime}\left(x_{j+1}\right)+K_{2}\left(x_{i}, x_{j}\right) u^{\prime}\left(x_{j}\right)\right] \mid, \\
\left|e_{2, n}\left(x_{i}\right)\right| & =\mid \sum_{j=0}^{n-1} \int_{x_{j}}^{x_{j+1}} \frac{\partial K_{1}}{\partial x}\left(x_{i}, t\right) u(t) d t-\frac{h}{2}\left[\frac{\partial K_{1}}{\partial x}\left(x_{i}, x_{j+1}\right) u\left(x_{j+1}\right)+\frac{\partial K_{1}}{\partial x}\left(x_{i}, x_{j}\right) u\left(x_{j}\right)\right] \\
& +\int_{x_{j}}^{x_{j+1}} \frac{\partial K_{2}}{\partial x}\left(x_{i}, t\right) u^{\prime}(t) d t-\frac{h}{2}\left[\frac{\partial K_{2}}{\partial x}\left(x_{i}, x_{j+1}\right) u^{\prime}\left(x_{j+1}\right)+\frac{\partial K_{2}}{\partial x}\left(x_{i}, x_{j}\right) u^{\prime}\left(x_{j}\right)\right] \mid .
\end{aligned}
$$


But for all $t \in\left[x_{j}, x_{j+1}\right]$,

$$
\begin{aligned}
& \left|\int_{x_{j}}^{x_{j+1}} K_{1}\left(x_{i}, t\right) u(t) d t-\frac{h}{2}\left[K_{1}\left(x_{i}, x_{j+1}\right) u\left(x_{j+1}\right)+K_{1}\left(x_{i}, x_{j}\right) u\left(t_{j}\right)\right]\right| \\
\leq & h\left[\max _{0 \leq i \leq n} \kappa_{0}\left(K_{1_{x_{i}}}, h\right)\|u\|_{X}+M_{1} \kappa_{0}(u, h)\right], \\
\leq & \left|\int_{x_{j}}^{x_{j+1}} K_{2}\left(x_{i}, t\right) u^{\prime}(t) d t-\frac{h}{2}\left[K_{2}\left(x_{i}, x_{j+1}\right) u^{\prime}\left(x_{j+1}\right)+K_{2}\left(x_{i}, x_{j}\right) u^{\prime}\left(x_{j}\right)\right]\right| \\
\leq & {\left[\max _{0 \leq i \leq n} \kappa_{0}\left(K_{x_{i}}, h\right)\|u\|_{X}+M_{2} \kappa_{0}\left(u^{\prime}, h\right)\right], } \\
\leq \quad & h\left[\int_{x_{j}}^{x_{j+1}} \frac{\partial K_{1}}{\partial x}\left(x_{i}, t\right) u(t) d t-\frac{h}{2}\left[\frac{\partial K_{1}}{\partial x}\left(x_{i}, x_{j+1}\right) u\left(x_{j+1}\right)+\frac{\partial K_{1}}{\partial x}\left(x_{i}, x_{j}\right) u\left(x_{j}\right)\right] \mid\right. \\
\leq & \left.\mid \int_{x_{j}}^{x_{j+1}} \frac{\partial K_{2}}{\partial x}\left(\frac{\partial K_{1_{x_{i}}}}{\partial x}, h\right)\|u\|_{X}+M_{1} \kappa_{0}(u, h)\right], \\
& h\left[\max _{0 \leq i \leq n} \kappa_{0}\left(\frac{\partial K_{x_{x_{i}}}}{\partial x}, h\right) d t-\frac{h}{2}\left[\frac{\partial K_{2}}{\partial x}\left(x_{i}, x_{j+1}\right) u^{\prime}\left(x_{j+1}\right)+\frac{\partial K_{2}}{\partial x}\left(x_{i}, x_{j}\right) u^{\prime}\left(x_{j}\right)\right] \mid\right.
\end{aligned}
$$

Which gives

$$
\begin{aligned}
\left|e_{1, n}\left(x_{i}\right)\right| & \leq(b-a)\left[M_{1} \max _{0 \leq i \leq n} \kappa_{0}\left(K_{1_{x_{i}}}, h\right)+M_{2} \max _{0 \leq i \leq n} \kappa_{0}\left(K_{2_{x_{i}}}, h\right)\right]\|u\|_{X} \\
& +(b-a)\left(M_{1}+M_{2}\right) \kappa_{1}(u, h), \\
\left|e_{2, n}\left(x_{i}\right)\right| & \leq(b-a)\left[M_{1} \max _{0 \leq i \leq n} \kappa_{0}\left(\frac{\partial K_{1_{x_{i}}}}{\partial x}, h\right)+M_{2} \max _{0 \leq i \leq n} \kappa_{0}\left(\frac{\partial K_{2_{x_{i}}}}{\partial x}, h\right)\right]\|u\|_{X} \\
& +(b-a)\left(M_{1}+M_{2}\right) \kappa_{1}(u, h) .
\end{aligned}
$$

Finally

$$
\begin{gathered}
\max _{0 \leq i \leq n}\left|e_{1, n}\left(x_{i}\right)\right|+\max _{0 \leq i \leq n}\left|e_{2, n}\left(x_{i}\right)\right| \\
\leq(b-a)\left[\begin{array}{c}
\left.M_{1} \max _{0 \leq i \leq n} \kappa_{1}\left(K_{1_{x_{i}}}, h\right)+M_{2} \max _{0 \leq i \leq n} \kappa_{1}\left(K_{2_{x_{i}}}, h\right)\right]\|u\|_{X} \\
+2(b-a)\left(M_{1}+M_{2}\right) \kappa_{1}(u, h) .
\end{array}\right.
\end{gathered}
$$

Then

$$
\left\|e_{n}\right\|_{\mathbb{R}^{2 n+2}} \leq(b-a) \sum_{p=1}^{2} M_{p}\left[\max _{0 \leq i \leq n} \kappa_{1}\left(K_{p_{x_{i}}}, h\right)\|u\|_{X}+2 \kappa_{1}(u, h)\right] .
$$


To demonstrate the effectiveness of our applied method and to prove the convergence of the approached solution, we will state the following theorem:

Theorem 4 Let $\varepsilon_{n}$, the error defined by:

$$
\varepsilon_{n}=\max _{0 \leq i \leq n}\left|u\left(x_{i}\right)-u_{i}\right|+\max _{0 \leq i \leq n}\left|u^{\prime}\left(x_{i}\right)-u_{i}^{\prime}\right|,
$$

Assuming that $|\lambda|>2(b-a)\left(M_{1}+M_{2}\right)$. Then $\lim _{n \rightarrow \infty} \varepsilon_{n}=0$.

ProOF we have

$$
\begin{aligned}
|\lambda|\left|u\left(x_{i}\right)-u_{i}\right| & \leq\left|e_{1, n}\left(x_{i}\right)\right|+(b-a)\left(M_{1}+M_{2}\right) \varepsilon_{n}, \\
|\lambda|\left|u^{\prime}\left(x_{i}\right)-u_{i}^{\prime}\right| & \leq\left|e_{2, n}\left(x_{i}\right)\right|+(b-a)\left(M_{1}+M_{2}\right) \varepsilon_{n} .
\end{aligned}
$$

For (19)-(20), we obtain:

$$
\varepsilon_{n} \leq \frac{b-a}{|\lambda|-2(b-a)\left(M_{1}+M_{2}\right)}\left\|e_{n}\right\|_{\mathbb{R}^{2 n+2}} .
$$

When $n \rightarrow \infty, \quad h=\frac{b-a}{n} \rightarrow 0$ and by theorem (3) $\lim _{n \rightarrow \infty}\left\|e_{n}\right\|_{\mathbb{R}^{2 n+2}}=0$, then $\lim _{n \rightarrow \infty} \varepsilon_{n}=0$.

\section{Numerical examples}

To show the effectiveness of the suggested methods, we apply this approach to the following numerical example:

$$
\forall x \in[0,1], \lambda u(x)=\int_{0}^{1} \frac{u(t)}{\sqrt{2+t^{3}+\frac{1}{1+x}}} d t+\int_{0}^{1} \frac{u^{\prime}(t)}{x+t^{4}+1} d t+f(x),
$$

where, $\lambda=4, u(x)=x^{2}$ and

$$
\begin{aligned}
& f(x)=4 x^{2}-\frac{\arctan \left(\frac{1}{\sqrt{x+1}}\right)}{\sqrt{x+1}}-\frac{2}{3}\left(\sqrt{3+\frac{1}{1+x}}-\sqrt{2+\frac{1}{1+x}}\right) . \text { We also have } \\
& \max _{0 \leq x, t \leq 1}\left(\left|K_{1}(x, t)\right|,\left|\frac{\partial K_{1}}{\partial x}(x, t)\right|\right) \leq \frac{4}{5} \text { and } \max _{0 \leq x, t \leq 1}\left(\left|K_{2}(x, t)\right|,\left|\frac{\partial K_{2}}{\partial x}(x, t)\right|\right) \leq 1 .
\end{aligned}
$$

Then, $|\lambda|=4>\frac{18}{5}$.

We give another example

$$
\forall x \in[0,1], \lambda u(x)=\int_{0}^{1} \frac{u(t)}{e^{x}+e^{t}} d t+\int_{0}^{1} \frac{u^{\prime}(t)}{1+x+e^{2 t}} d t+f(x),
$$

where, $\lambda=2, u(x)=e^{x}$ and 
Table 1. The error between the exact and approximation solution of equation (22)

\begin{tabular}{|c|c|c|c|c|}
\hline $\mathrm{n}$ & 10 & 100 & 500 & 1000 \\
\hline $\max _{0 \leq i \leq n}\left|u\left(x_{i}\right)-u_{i}\right|$ & $7.0610 \mathrm{e}-05$ & $7.0506 \mathrm{e}-07$ & $2.8202 \mathrm{e}-08$ & $7.0505 \mathrm{e}-09$ \\
\hline $\max _{0 \leq i \leq n}\left|u^{\prime}\left(x_{i}\right)-u_{i}^{\prime}\right|$ & $1.6370 \mathrm{e}-04$ & $1.6353 \mathrm{e}-06$ & $6.5410 \mathrm{e}-08$ & $1.6352 \mathrm{e}-08$ \\
\hline$\varepsilon_{n}$ & $2.3431 \mathrm{e}-04$ & $2.3403 \mathrm{e}-06$ & $9.3612 \mathrm{e}-08$ & $2.3403 \mathrm{e}-08$ \\
\hline time (seconds) & 0.023258 & 0.049113 & 0.774680 & 3.641881 \\
\hline
\end{tabular}

$f(x)=\log \left(e^{x}+1\right)-\log \left(e^{1}+e^{x}\right)+2 e^{x}-\arctan \left(\frac{e^{1}}{\sqrt{x+1}}\right)-\frac{\arctan \left(\frac{1}{\sqrt{x+1}}\right)}{\sqrt{x+1}}$. We prove that

$$
\max _{0 \leq x, t \leq 1}\left(\left|K_{1}(x, t)\right|,\left|\frac{\partial K_{1}}{\partial x}(x, t)\right|\right) \leq \frac{1}{3} \text { and } \max _{0 \leq x, t \leq 1}\left(\left|K_{2}(x, t)\right|,\left|\frac{\partial K_{2}}{\partial x}(x, t)\right|\right) \leq \frac{1}{2} .
$$

Then, $|\lambda|=2>\frac{10}{6}$.

Table 2. The error between the exact and approximation solution of equation (23)

\begin{tabular}{|c|c|c|c|c|}
\hline $\mathrm{n}$ & 10 & 100 & 500 & 1000 \\
\hline $\max _{0 \leq i \leq n}\left|u\left(x_{i}\right)-u_{i}\right|$ & $1.0479 \mathrm{e}-04$ & $1.0468 \mathrm{e}-06$ & $4.1871 \mathrm{e}-08$ & $1.0468 \mathrm{e}-08$ \\
\hline $\max _{0 \leq i \leq n}\left|u^{\prime}\left(x_{i}\right)-u_{i}^{\prime}\right|$ & $5.8898 \mathrm{e}-05$ & $5.8873 \mathrm{e}-07$ & $2.3549 \mathrm{e}-08$ & $5.8873 \mathrm{e}-09$ \\
\hline$\varepsilon_{n}$ & $1.6368 \mathrm{e}-04$ & $1.6355 \mathrm{e}-06$ & $6.5420 \mathrm{e}-08$ & $1.6355 \mathrm{e}-08$ \\
\hline time (seconds) & 0.071628 & 0.048551 & 0.502111 & 3.834747 \\
\hline
\end{tabular}

We applied the method proposed to approximate the solution of (22) and (23), and we calculated the error between the approximation and exact solution which is given by:

$$
\varepsilon_{n}=\max _{0 \leq i \leq n}\left|u\left(x_{i}\right)-u_{i}\right|+\max _{0 \leq i \leq n}\left|u^{\prime}\left(x_{i}\right)-u_{i}^{\prime}\right| .
$$

Table 1 presented the numerical results of equation (22) and Table 2 presented the numerical results of equation (23).

For better observation on the effectiveness of our proposed method, we plot the approximate and exact solutions of (22) in Figure 1 and the approximate and exact solutions of (23) in Figure 2 in different collocation points $x_{i}$. Figures 1 and 2 clearly prove the accuracy of our numerical method proposed in this paper.

\section{Conclusion}

In this article, we have studied a a different integro-differential equation than the existing ones. We proposed the Nyström technique to obtain an approach solution of linear Fredholm integro-differential equation. This method helped us to reformulate an equation to a linear system by block. Before applying this estimation, we posed some assumptions at the beginning to provide the existence and uniqueness of the 


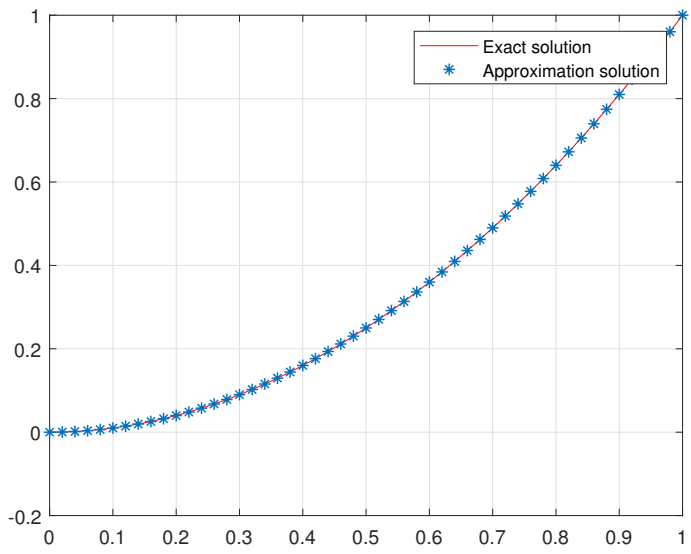

Fig. 1. The approximate and exact solutions with the Nyström method for equation (22), $n=50$

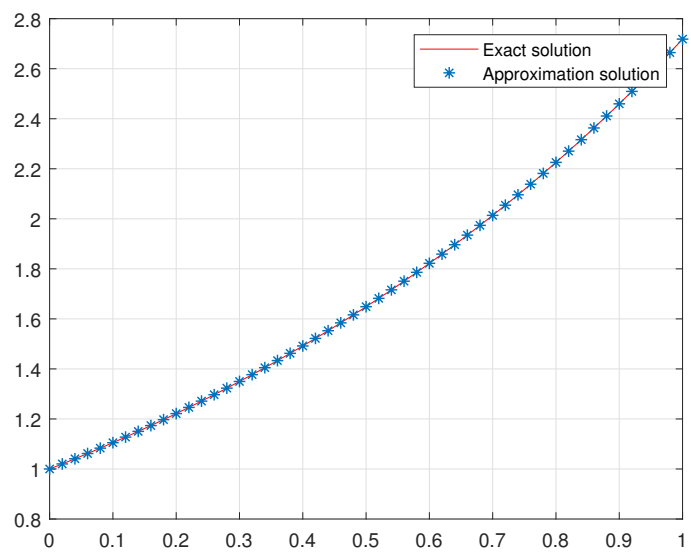

Fig. 2. The approximate and exact solutions with the Nyström method for equation (23), $n=50$

solution on the one hand and on the other hand, to affirm that the approximate solution converges to the exact solution in the space $C^{1}[a, b]$. We have constructed an error bound of the Nyström method for a function in $C^{1}[a, b]$. At the end, the comparison of the numerical results has shown that the method presented is an interesting tool for finding a numerical solution to the linear Fredholm's integro-differential equation.

As perspectives, we will apply the iterative methods to avoid the calculation of inverse matrix of system (16), especially when the size of this matrix is large. Other works in progress are developed around these kind of equations. We are developing an analytical and numerical study on the equation (1) in the Sobolev space $W^{1,1}(a, b)$. Also, we have the possibility to apply a new projection method based on Legendre polynomials in the Sobolev space $H^{1}(a, b)$ or cubic b-spline functions and we take as a consideration all different cases of the kernels. 


\section{Acknowledgments}

We extend our sincere thanks to Redwan Geutatlia.

\section{References}

[1] Lakshmikantham, V. (1995). Theory of Integro-Differential Equations (Vol. 1). CRC Press.

[2] Jerri, A. (1999). Introduction to Integral Equation with Application. John Wiley and Sons.

[3] Singh, H., Dutta, H., \& Cavalcanti, M.M. (2021). Topics in Integral and Integro-Differential Equations, Studies in Systems. Springer.

[4] Zemyan, S.M. (2012). The Classical Theory of Integral Equations. Birkhäuser Basel, Springer, New York.

[5] Yusufoglu, E. (2007). An efficient algorithm for solving integro-differential equations system. Applied Mathematics and Computing, 192, 51-55.

[6] Singh, C.S., Singh, H., Singh, V.K., \& Singh, Om, P. (2016). Fractional order operational matrix methods for fractional singular integro-differential equation. Applied Mathematical Modelling, 40, 10705-10718.

[7] Singh, C.S., Singh, H., Singh, S., \& Kumar, D. (2018). An efficient computational method for solving system of nonlinear generalized Abel integral equations arising in astrophysics. Physica A, 525, 1440-1448.

[8] Singh, H., Baleanu, D., Srivastava, H.M., Dutta, H., \& Jha, N.K. (2020). Solution of multidimensional Fredholm equations using Legendre scaling functions. Applied Numerical Mathematics, 150, 313-324.

[9] Tiwari, S., Pandey, R.K., Singh, H., \& Singh, J. (2020). Embedded pseudo-Runge-Kutta methods for first and second order initial value problems. Science \& Technology Asia, 25(1), 128-141.

[10] Pandey, R.K., \& Singh, H. (2020). An efficient numerical algorithm to solve volterra integral equation of second kind. Topics in Integral and Integro-Differential Equations. Studies in Systems, Decision and Control, 340, 215-228.

[11] Shanga, X., \& Hanb, D. (2010). Application of the variational iteration method for solving nth-order integro-differential equations. Journal of Computianal Applied Mathematics, 234, 1442-1447.

[12] Mennouni, A. (2012). A projection method for solving Cauchy singular integro-differential equations. Applied Mathematics Letters, 25, 986-989.

[13] Atkinson, K., \& Han, W. (2001). Theoretical Numerical Analysis: A Functional Analysis Framework. Springer-Verlag, New York.

[14] Guebbai, H., Aissaoui, M.Z., Debbar, I., \& Khalla, B. (2014). Analytical and numerical study for an integro-differential nonlinear volterra equation. Applied Mathematics and Computation, 299, 376-373.

[15] Guebbai, H., Lemita, S., Segni, S., \& Merchela, W. (2020). Difference derivative for an integro-differential nonlinear volterra equation. Vestnik Udmurtskogo Universiteta Matematika Mekhanika Komp'uternye Nauki, 30(2), 176-188.

[16] Zhou, H., \& Wang, Q. (2019). The Nyström method and convergence analysis for system of Fredholm integral Equations. Fundamental Jornal of Applied Mathematics, 2(1), 28-32.

[17] Segni, S., Ghiat, M., \& Guebbai, H. (2019). New approximation method for Volterra nonlinear integro-differential aquation. Asian-European Journal of Mathematics, 12(1), 1950016.

[18] Ghiat, M., Guebbai, H., Kurulay, M., \& Segni, S. (2020). On the weakly singular integrodifferential nonlinear Volterra equation depending in acceleration term. Computational and Applied Mathematics, 39(2), 206. 
[19] Salah, S., Guebbai, H., Lemita, S., \& Aissaoui, M.Z. (2019). Solution of an integro-differential nonlinear equation of Volterra arising of earthquake model. Boletim da Sociedade Paranaense de Matemática, 1-14.

[20] Lemita, S., \& Guebbai, H. (2019). New process to approach linear Fredholm integrale quations defined on large interval. Asian-European Journal of Mathematics, 12(1), 1950009.

[21] Touati, S., Lemita, S., Ghiat, M., \& Aissaoui M.Z. (2019). Solving a non-linear Volterra-Fredholm integro-differentail equation with weakly singular kernels. Fasciculi Mathematics, $62,155-168$

[22] Ghiat, M., \& Guebbai, H. (2018). Analytical and numerical study for an integro-differential nonlinear volterra equation with weakly singular kernel. Computational and Applied Mathematics, 37(4), 4661-4974.

[23] Davies, E.B. (2007). Linear Operators and their Spectra. Cambridge University Press, Cambridge.

[24] Ahues, M., Largillier, A., \& Limaye, B.V. (2001). Spectral Computations for Bounded Operators. Chapman and Hall/CRC, Boca Raton.

[25] Quarteroni, A., Sacco, R., \& Saleri, F. (2004). Méthodes Numériques: Algorithmes, analyse et applications. Springer. 